\title{
Retos y oportunidades en la enseñanza del español para extranjeros en una universidad mexicana
}

\author{
Challenges and Opportunities in Teaching Spanish to Foreigners at a Mexican \\ University \\ Virna Velázquez-Vilchis ${ }^{a}$ Edgar E. García-Ponce ${ }^{b}$
}

\begin{abstract}
:
Spanish has recently become a prominent language in the world. According to the 2020 report of the Cervantes Institute, more than 585 million people speak Spanish as their mother tongue and as second or foreign language. Native speakers reach almost 489 million. This language is the second most spoken mother tongue by number of speakers, and in the global population there are $7.5 \%$ of Spanish speakers. Today more than 22 million students study it as a foreign language. However, there are several factors which limit foreigners from coming to Mexico to learn the language. At the heart of the matter lies the fact that Mexican Spanish our variant is not a variety widely recognized in other countries. Other examples are the lack of materials to teach our variant, but also the situation of teaching Spanish to foreigners in Mexican universities. The aim of the present article is threefold. First, it describes the current situation of Spanish teaching for foreigners in a university in central Mexico. Second, it evaluates some of the language policies to promote the teaching and learning of this language. It finally explores the challenges that the stakeholders have perceived in this context, concerning teaching, and learning practices with a view to strengthening the teaching of Spanish at this institution.
\end{abstract}

Keywords:

Teaching, Public University, language planning

\section{Resumen:}

Recientemente el español se ha vuelto una predominante en el mundo. De acuerdo con el informe 2020 del Instituto Cervantes, más de 585 millones de personas lo hablan como lengua nativa, segunda o extranjera, siendo los usuarios nativos alrededor de 489 millones. El español es la segunda lengua materna de mayor presencia por número de hablantes y en la población mundial existen $7.5 \%$ de hispanohablantes. Hoy en día, más de 22 millones de alumnos estudian español como lengua extranjera. Sin embargo, existe una serie de factores que limitan que los extranjeros decidan venir a México para aprender la lengua. Algunos de los factores obedecen a que nuestra variante no es bien vista en otros países, a la carencia de materiales pertinentes para enseñar nuestra variante, pero también a la situación de la enseñanza de español para extranjeros en las universidades mexicanas. El presente artículo tiene tres objetivos. Primero, describir la situacion de la enseñanza de español como lengua extranjera (ELE) de una universidad en el bajío de México. Segundo, evaluar algunas de las políticas lingüísticas que se tienen actualmente al interior de esta universidad a fin de fortalecer aquellas acciones que les permite mantener un número de extranjeros. Tercero, hacer una exploración de los retos para que, a corto y mediano plazo, los involucrados puedan fortalecer la enseñanza y el aprendizaje del español como lengua extranjera en su institución.

\section{Palabras Clave:}

Enseñanza, Universidad Pública, planificación lingüistica

\footnotetext{
${ }^{a}$ Virna Velázquez Vilchis, Facultad de Lenguas, Universidad Autónoma del Estado de México, https://orcid.org/0000-0002-5630-9360, Email: vvir71@gmail.com

${ }^{b}$ Edgar E. García-Ponce, Departamento de Lenguas de la División de Ciencias Sociales y Humanidades, Universidad de Guanajuato,
} https://orcid.org/0000-0002-5414-3862, Email: ee.garcia@ugto.mx 


\section{INTRODUCCIÓN}

En la actualidad, el español tiene 470 millones de nativo hablantes (Centro Virtual Cervantes, 2018). De acuerdo con los cursos que imparte el Instituto Cervantes en el mundo, la matrícula se ha incrementado año con año, produciendo así una tendencia de crecimiento de la demanda de los cursos de español. Cuando entramos a sitios de internet como: todoELE (Suárez García, 2021), Edinumen o cervantes.es (Instituto Cervantes), además de encontrar ofertas de clases, podemos obtener información sobre capacitaciones para docentes, congresos, asociaciones, certificaciones, ofertas de trabajo y materiales de enseñanza, por lo que es lógico pensar que, cada vez más, la industria de la enseñanza del español como lengua extranjera (ELE) se fortalece. No obstante, en México se trata de un área poco explorada por las instituciones educativas públicas; lo que ofrece múltiples oportunidades debido a las condiciones educativas nacionales.

Por otro lado, las instituciones que se dedican a normalizar y homologar el quehacer de la enseñanza de español para extranjeros en nuestro país son pocas. Quizás sea el Centro de Enseñanza de Español para Extranjeros (CEPE) de la Universidad Nacional Autónoma de México (UNAM) el caso más reconocido en México. Esta institución certifica a nivel nacional la enseñanza de español a extranjeros, pero las actividades que desarrolla no necesariamente cubren las necesidades de otras instituciones educativas públicas en el país. Para fortalecer el quehacer sobre la enseñanza de español en dichas instituciones se debe conocer primero la situación lingüística y pedagógica que viven, a fin de poder tomar decisiones académicamente adecuadas y con base en necesidades locales, es decir, con base en una planificación lingüística pertinente (Kaplan y Baldauf, 1997). La planificación lingüística, de acuerdo con Tollefson (1994), se define como la búsqueda de estrategias para satisfacer necesidades lingüísticas de una comunidad y que sirven para diversos propósitos.

Con base en lo anterior, el objetivo de este artículo es presentar los resultados de un proyecto de investigación que se llevó a cabo en la Universidad de Guanajuato en 2020. El objetivo de esta investigación es analizar la situación de la enseñanza de ELE en esta institución para comprender las condiciones que viven $\mathrm{y}$, en su caso, proporcionar esta información a la comunidad, a fin de que se puedan establecer estrategias de mejora. Específicamente, el estudio busca explorar los aciertos y retos que enfrentan en torno a la enseñanza del español para extranjeros.

\section{LOS EXTRANJEROS EN MÉXICO}

De acuerdo con datos del INEGI (2020), hay mayor movimiento migratorio hacia nuestro país el cual se ve reflejado en el porcentaje considerable de inmigrantes que se sienten atraídos por México. Los extranjeros que llegan al país se quedan para estudiar, trabajar e incluso vivir de forma permanente. Según esta institución, los residentes inmigrantes en el país pasaron de cerca de 101 mil a casi medio millón respectivamente. Es decir, en casi un siglo de levantamientos censales en México, el incremento que registró esta población equivale aproximadamente a 392 mil efectivos, que representan $79.5 \%$ de los extranjeros residentes en México en el año 2000. El documento Los extranjeros en México del INEGI (2007) señala que la gran mayoría de los extranjeros residentes en México nacieron en los EUA y su lengua materna es el español. Sin embargo, los extranjeros residentes en México cuya lengua no es el español son los franceses y los canadienses que representan $1.2 \%$ de esta población respectivamente, los italianos $(0.8 \%)$, los japoneses $(0.6 \%)$, los ingleses y los brasileños $(0.5 \%)$, los alemanes y los chinos $(0.4 \%)$, los rusos $(0.3 \%)$, los libaneses $(0.2 \%)$ y otras nacionalidades no especificadas que representan $6.1 \%$ de la población en México; es decir, cerca del 12\% de la población de extranjeros en México tiene otro idioma como primera lengua.

Ruiz Zambrana (2009) recalca que el español, además de ser el vehículo de comunicación en relaciones comerciales, laborales y diplomáticas, es uno de los tres idiomas de trabajo de la ONU y uno de los seis idiomas para reuniones oficiales, junto al árabe, el chino y el ruso. Ahora bien, la presencia de la lengua española como lengua oficial en casi la mayoría de los países del continente americano, su extensión geográfica más allá de este continente, la fuerte presencia latina en los Estados Unidos, el peso del español en el internet (con aproximadamente 22 millones de usuarios y 120 millones de páginas web en español, es la tercera lengua más usada en el internet y su uso crece cada vez más en cuanto al número de personas no hablantes nativas que desean aprenderla. Según datos del Instituto Cervantes, el vasto incremento de negocios internacionales debido a la globalización de nuestro mundo, entre otras varias razones, hacen del español una lengua muy demandada en el campo de la enseñanza de lenguas extranjeras en países no hispanos de América y de Europa, principalmente.

Pérez Velasco y González Freire (2008) entrevistaron a un grupo de estudiantes de español como lengua extranjera provenientes de diferentes nacionalidades. Su objetivo era conocer las razones por las cuales les interesaba aprender español en México. Específicamente, los encuestados respondieron que el saber otra lengua, aparte del inglés, les otorgaba una ventaja sobre sus compañeros, sobre todo por cuestiones económicas. Los estadounidenses consideraban que el aprender español como lengua extranjera era la mejor opción por la gran población hispana que vive en su país, y por ser vecinos de México. Los asiáticos, por su lado, mencionaron los aspectos de negocios y las ventajas que les brinda el hablar español con otras personas. Por su parte, el grupo de los canadienses, holandeses y suizos 
pensaron más en los viajes y en el acercamiento a la cultura y a la gente de Latinoamérica.

\section{UNIVERSIDADES MEXICANAS PÚBLICAS PARA LA ENSEÑANZA Y APRENDIZAJE DEL ESPAÑOL PARA EXTRANJEROS}

Si bien existe un número significativo de escuelas privadas en donde estudiar español en el país, son menos las universidades públicas que se especializan en ello. Muestra de ello es que muchas universidades mexicanas ofrecen cursos de español para extranjeros en sus páginas oficiales; sin embargo, aunque ofertan este tipo de cursos, no cuentan con estudiantes para mantener un programa permanente de alumnos extranjeros.

La mayoría de las universidades públicas, además de ofrecer las clases de español, brindan apoyo a sus alumnos para el hospedaje, en algunos casos, el transporte, además de excursiones académicas o culturales y actividades extracurriculares donde los extranjeros pueden convivir con personas nativas del español en inmersión.

Como hemos ya señalado, consideramos que la institución líder en la enseñanza del ELE en nuestro país es el CEPE de la UNAM. Desde su fundación en 1921, el CEPE ha ofertado cursos de diversa naturaleza: español, arte, literatura, cultura, historia mexicana, los que se ofrecen de manera permanente a lo largo de los ciclos escolares. También, cuenta con exámenes para la certificación internacional de la lengua española, participa como creador del SIELE junto con el Instituto Cervantes y las Universidades de Salamanca, y Buenos Aires. De igual forma cuenta con programas académicos para la formación de docentes de español como lengua extranjera, cursos extra-clase, tanto académicos como de contenido experiencial, para los alumnos y visitas guiadas. La sede principal se ubica en Ciudad Universitaria (CU), pero la institución cuenta con otras sedes en el país, como la sede en Polanco y en Taxco, Guerrero, y otras tantas en el extranjero, por ejemplo, las sedes de Chicago, Londres, París y la de Sudáfrica.

En lo que respecta a formación en el área de la enseñanza del ELE, el CEPE ofrece dos diplomados: el Diplomado Básico en Enseñanza de Español como Lengua Extranjera (DIBEELE) y el Diplomado Avanzado en Enseñanza de Español como Lengua Extranjera (DIAPELE), así como la Especialización en Enseñanza de Español como lengua Extranjera a distancia (EEELE) junto con la Escuela Nacional de Lenguas, Lingüística y Traducción (ENALLT). La UNAM cuenta, además, con la Licenciatura en Enseñanza de Español como Lengua Extranjera (LICEL), Modalidad a distancia en el Sistema de Universidad Abierta. La licenciatura permite que los alumnos se especialicen en la enseñanza de una de las siguientes lenguas: alemán, español, francés, inglés o italiano. Lo que brinda la posibilidad de especializarse en el español, aunque no necesariamente brinda un énfasis para enseñanza a extranjeros.

La Universidad Veracruzana, campus Xalapa, cuenta con más de 60 años en la enseñanza del español como lengua extranjera, a través de la Escuela para Estudiantes Extranjeros (EEE) en donde proporciona cursos de español por semestre e intensivos. También oferta talleres, cursos de cultura, historia, literatura, y además se encarga de involucrar a los estudiantes extranjeros en actividades como el servicio voluntario, talleres artísticos, visitas y viajes culturales. Por último, esta universidad es, además, centro examinador autorizado SIELE, del Instituto Cervantes y ofrece el Diplomado en didáctica del español como lengua extranjera (DELE), modalidad presencial y virtual, dirigido a docentes de español como lengua materna o como lengua extranjera que desean profesionalizarse.

Por otro lado, en la región del occidente mexicano, se encuentra la Universidad de Guadalajara, la cual posee el Programa de Español Para Extranjeros (PEPE). El PEPE se articula en seis cursos de español intensivo y cada nivel es de 40 horas. Asimismo, la Universidad de Guadalajara brinda cursos de historia y cultura mexicana, talleres, excursiones y actividades culturales de inmersión. Además, el Centro de Investigación y Certificación del Español como Lengua Extranjera y Materna (CICELEM), adscrito al Departamento de Letras de la División de Estudios Históricos y Humanos del Centro Universitario de Ciencias Sociales y Humanidades regula la investigación, difusión y docencia del ELE e imparte el Diplomado: Enseñanza del Español como Segunda Lengua.

En la zona del bajío, encontramos a la Universidad Autónoma de Querétaro, quienes a través de la Facultad de Lenguas y Letras ofrecen cursos de español para extranjeros. Este organismo cuenta con 35 años de experiencia y apertura cursos curriculares y no curriculares, con distinta carga horaria a lo largo del año escolar. Finalmente, también en esta misma zona, la Universidad de Guanajuato (UG), el contexto del presente estudio cuenta con la Licenciatura en Enseñanza de Español como Segunda Lengua, en su campus Guanajuato. Esta licenciatura se oferta para aquellos interesados en adentrarse en los confines de la didáctica del ELE. Además, la universidad proporciona cursos cortos y permanentes de español como segunda lengua durante el año escolar, así como talleres de baile, literatura, cultura, entre otros.

Indudablemente, lo anterior muestra que las universidades mexicanas han hecho esfuerzos para fomentar la enseñanza del español como lengua extranjera y para promover nuestra riqueza cultural. Sin embargo, después de haber hecho una búsqueda en internet de las principales páginas oficiales de las universidades públicas mexicanas, con respecto a las posibilidades que brindan para la enseñanza del español para extranjeros, podemos decir que, hasta el momento de esta publicación, existe poca información que dé cuenta sobre la situación de los programas y 
cursos del español para extranjeros en nuestras universidades públicas. Esto deja entrever la necesidad de indagar con cuidado las condiciones que se viven en las instituciones para entender sus fortalezas y los retos que se deben atender para promover la enseñanza del español a extranjeros y continuar incrementando el número de personas que eligen a nuestro país como destino para aprender la lengua y la cultura. Para contribuir a este propósito, enseguida se abordan los pormenores de la investigación que se hizo durante el año 2020.

\section{EL ESTUDIO}

Para poder mejorar la oferta de la enseñanza de ELE, conviene conocer cuál es el impacto general en la población y contar con una etnografía de la comunicación que describa las condiciones generales $\mathrm{y}$, de esta manera, profundizar $\mathrm{y}$ atender la problemática detectada. Actualmente, la Universidad de Guanajuato no cuenta con un estudio sistematizado ni información cuantificada sobre las diferentes estrategias de mejora que se han implementado por parte de directivos y docentes. Tampoco se sabe el impacto que dichas estrategias ha tenido en la población estudiantil. Para las universidades mexicanas es imperante este tipo de información, pues debemos pasar por evaluaciones nacionales de calidad académica si queremos acceder a recursos federales y rankings de calidad.

Es indudable que la Universidad de Guanajuato goza de prestigio en Latinoamérica, ya que se encuentra ubicada entre las 100 mejores universidades de Latinoamérica y del Caribe del Ranking Iberoamericano SIR 2012. En México, de las 264 universidades evaluadas se ubicó entre las diez mejores del país, por lo que podemos decir que se distingue como de excelencia a nivel nacional. Entre los interesados en perfeccionar su dominio del español, también se percibe a la institución con prestigio, tanto por su ubicación geográfica como por los programas que ahí se ofrecen. Sobre todo, por los cursos de español para extranjeros que imparte y porque es una de las pocas universidades públicas a nivel nacional que cuenta con una licenciatura en español para no nativos de la lengua. La universidad oferta cada semestre cursos de español para extranjeros, así como su programa de licenciatura para futuros profesores de español para no nativos: la Licenciatura en Enseñanza del Español como Segunda Lengua (LEES).

Como muchas instituciones de educación superior en México, la universidad se ha visto afectada por la inseguridad en los años recientes, lo que ha hecho que el número de estudiantes extranjeros vaya a la baja. Empero, existe una serie de circunstancias al interior del organismo, que podría ayudar a que este número se incrementara. Y es precisamente ahí donde esta investigación contribuye.

Aunque al inicio de la investigación se recolectaron algunos datos, por las condiciones de pandemia en 2020, la información tuvo que ser recabada a través de medios electrónicos con ayuda del internet. Los extranjeros que se encontraban estudiando en la institución regresaron a sus países de origen, lo que nos obligó a aplicar el cuestionario por medios electrónicos. Lo anterior tuvo un impacto significativo en la disminución del número de informantes extranjeros originalmente planeados, el cual era de 50 alumnos. Sin embargo, solo se pudo contactar a diez. Esto representa una quinta parte $(20 \%)$ del número total aproximado de estudiantes extranjeros que asisten a la institución cada semestre, pues semestralmente se recibe un promedio de 50 alumnos extranjeros.

Por otro lado, los estudiantes de la LEES fueron confinados, lo que nos llevó a aplicar el instrumento también por medios electrónicos. Desafortunadamente, y por la incertidumbre inicial en el sistema educativo, no contamos con una cantidad significativa de respuestas como planeamos inicialmente. De una población total en el primer semestre 2020 de 83 alumnos, obtuvimos respuestas de solo 13 participantes, lo que representa aproximadamente el $15 \%$ del total de la población al momento de la aplicación del instrumento. Sin embargo, por tratarse de una comunidad pequeña en este contexto, consideramos que el número de respuestas es válido, más no generalizable. Además, no se pretende generalizar los resultados que surjan del estudio, sino entender las opiniones y experiencias subjetivas de los participantes (Dörnyei, 2007) en cuanto a procesos de enseñanza del español en este contexto particular.

Para llevar a cabo nuestra recolección de datos, se siguieron principios de la investigación mixta (Creswell, 2014) que implica combinar los enfoques cuantitativo y cualitativo en un mismo estudio. Los instrumentos que se aplicaron fueron de dos tipos: un cuestionario con preguntas cerradas y abiertas, y una entrevista guiada a distintas personas involucradas en esta área. Enseguida presentamos una descripción detallada de los mismos.

\section{INSTRUMENTOS}

Para obtener los datos, se aplicaron y analizaron dos cuestionarios diagnósticos con preguntas cerradas y abiertas. Uno, para alumnos extranjeros que tomaron cursos de español en la institución en el último año y otro, para estudiantes de la LEES. El objetivo en ambos casos era permitir que los alumnos indicaran las fortalezas en su paso por la institución, así como detectar necesidades en términos de qué se requiere para enriquecer la enseñanza, el diseño de materiales, y en general las oportunidades que la universidad les ofrece, así como verter sus sugerencias para mejorar sus condiciones de enseñanza y aprendizaje.

Ambos cuestionarios cuentan con seis secciones. Para los alumnos extranjeros, la sección uno incluye información personal como edad, sexo y país de procedencia. La sección dos 
averigua los años que lleva el estudiante aprendiendo español, así como las razones para aprender esta lengua. La siguiente sección sondea las razones que tuvo para decidir hacer su movilidad en esta Universidad en México y cómo supo de la misma. La sección cuatro es un conjunto de oraciones para saber el grado de satisfacción que tiene el alumno en términos del profesor, el contenido académico, materiales que usan los docentes, horarios de los cursos, los apoyos que reciben por parte de la institución (becas), instalaciones y apoyo para hospedaje. Las opciones que se dieron eran cinco: muy satisfecho, satisfecho, indiferente, poco satisfecho y nada satisfecho.

Posteriormente viene una sección para dar su opinión general sobre el proceso de enseñanza - aprendizaje en la institución y recomendaciones que puedan hacer para la mejora. Finalmente, una sección para saber si el alumno piensa certificarse en la lengua o si cuenta con algún tipo de certificación. La certificación, como veremos más adelante, es un área de oportunidad, pues si los alumnos desean certificarse pueden solicitar cursos con estos propósitos. Al final se deja un apartado de comentarios por si el alumno quiere agregar alguna otra información adicional.

Al igual que el cuestionario de estudiantes extranjeros, el cuestionario para estudiantes de la LEES contiene seis secciones. La primera sección contiene información personal como edad y sexo, la segunda sección indaga las razones para estudiar la licenciatura y cómo supo de la carrera. En la siguiente sección se sondea el conocimiento del plan de estudios por parte del alumno. La cuarta sección aborda el grado de satisfacción del alumno en término de profesores, contenidos académicos, materiales, horarios, becas, instalaciones y prácticas sobre la enseñanza de español. Al igual que el cuestionario anterior, se incluyen cinco opciones: muy satisfecho, satisfecho, indiferente, poco satisfecho y nada satisfecho. También se incluye un espacio para otros comentarios. La sección cinco es la opinión del alumno en cuanto a su sentir sobre la preparación al campo laboral al momento de egreso y recomendaciones para la mejora de dicha preparación. Finalmente, la certificación en el área del español por parte del alumno para ejercer como docente. Al final se deja un apartado de comentarios por si quiere agregar alguna información extra.

Se aplican también nueve entrevistas guiadas a profesores, coordinadores de la licenciatura y de los cursos de español, así como a administrativos, que tienen que ver con las gestiones de los cursos. Cada entrevista tuvo una duración aproximada de una hora, posteriormente fueron transcritas y analizadas. Estas entrevistas tienen el objetivo de conocer con mayor detalle la situación que viven los diferentes responsables de la enseñanza en la institución.

Las preguntas tratan diferentes áreas como la formación académica, funciones que se llevan a cabo, su grado de participación en la planeación del curriculum, su conocimiento sobre el mismo, los requisitos que debieron cubrir para formar parte del programa, las fortalezas que existen, así como las debilidades que perciben. Todos los participantes habían trabajado en el último año impartiendo u organizando cursos relacionados a la enseñanza del español para extranjeros.

Para la confiabilidad y validez de los instrumentos se contó con tres expertos jueces, así como con un grupo de alumnos extranjeros que no formaron parte de la investigación. Una vez recabados sus comentarios y sugerencias se hicieron los ajustes pertinentes.

\section{LA SITUACIÓN DE LA ENSEÑANZA DEL ELE EN LA INSTITUCIÓN}

La exigencia institucional marca que todos los programas educativos de la Universidad de Guanajuato se acoplen al nuevo Modelo Educativo como fue señalado en la modificación al Reglamento de Modalidades de los Planes de Estudio (aprobada por el Consejo General Universitario mediante el acuerdo CGU2013-O3-03 del 29 de agosto del 2013, p. 12), donde "se pretende que los programas educativos que se imparten, o lleguen a impartirse, en la Universidad otorguen los créditos académicos respectivos a partir del trabajo del estudiante [...], promoviendo y reconociéndole su trabajo autónomo como sugiere el Modelo Educativo institucional, además de establecer condiciones para una mayor movilidad estudiantil, toda vez que los parámetros de reconocimiento al trabajo del estudiante y la consecuente asignación de créditos es congruente con la tendencia predominante en Europa, Asia y la mayor parte de América Latina". La misma modificación incluye algunas reformas al Modelo Educativo, anteriormente expedido en el 2008, que adecuaron el documento al PLADI 2010-2020.

Como todas las universidades públicas, el plan de desarrollo institucional de esta institución contempla la internacionalización de la enseñanza y el aprendizaje. En otro de sus apartados se describen las categorías centrales; para nosotros, la de mayor interés señala que la universidad tiene que fortalecerse e incrementar las oportunidades de internacionalización.

Una forma de hacerlo es a través de la preparación de sus estudiantes al campo laboral, que sus egresados cuenten con las herramientas teóricas, laborales y practicas necesarias para llevar a cabo su trabajo y que puedan competir en el ámbito internacional. Otra manera es a través de la oferta de cursos de español para no hispanohablantes. Por otro lado, la institución reconoce la importancia de contar con profesionales capacitados, competentes tanto en una lengua extranjera como en el español para instruir a aquellos cuyo conocimiento del español es limitado. En este apartado discutimos hasta qué punto se logra este objetivo. 


\section{LOS ALUMNOS EXTRANJEROS}

Se pudieron obtener diez respuestas de extranjeros de distintas nacionalidades: tres estadounidenses, un japonés y seis coreanos. Las edades oscilaban entre los 21 a los 25 años. Del total de respuestas, siete fueron hombres y tres mujeres.

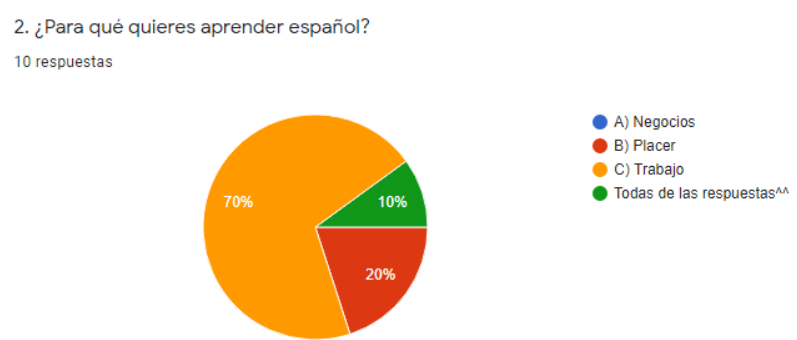

Gráfica 1. Razones de los extranjeros para aprender español

Como se observa en la Gráfica 1, para los alumnos extranjeros, el español es necesario por razones laborales, por placer y un alumno indicó que tanto por negocios, como por placer y trabajo. Los extranjeros decidieron estudiar en esa institución por recomendación, la mitad de ellos se enteró de esta universidad por medio de su propia institución, también por el prestigio que tiene y por amigos o familiares que radican en esa ciudad.

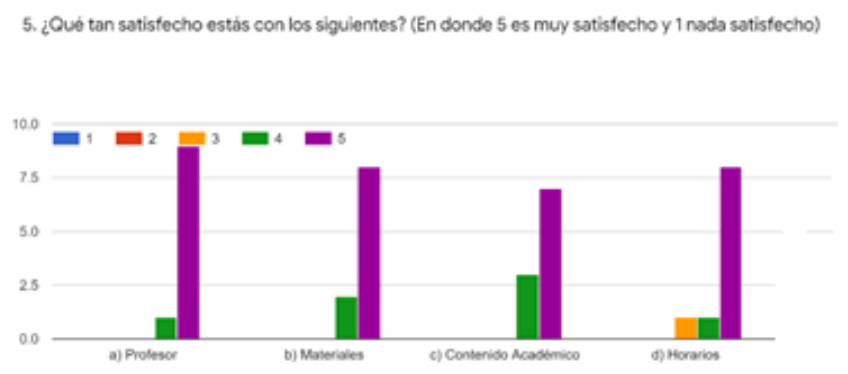

Gráfica 2. Grado de satisfacción durante su estancia

Los estudiantes dijeron estar al $100 \%$ satisfechos o muy satisfechos de las clases que recibieron durante su estancia, pese a que esta institución no cuenta con sus propios materiales institucionales. Los estudiantes también se sintieron satisfechos o muy satisfechos y todos expresaron satisfacción con los contenidos académicos. En cuanto a los apoyos que recibieron durante su estancia en términos de ayuda económica o becas, instalaciones y apoyo para hospedajes, se sintieron en su mayoría muy satisfechos. De estas respuestas podemos señalar que la universidad cuida que los extranjeros que reciben tengan muy buena atención académica y personal durante su estadía. Algunas sugerencias vertidas fueron la ampliación de horarios para clase y más comunicación con la parte administrativa.
El $90 \%$ de los estudiantes extranjeros no cuenta con una certificación y $80 \%$ planea certificarse en el corto plazo. Este porcentaje de alumnos sin certificarse es una oportunidad para la universidad, pues si se ofrecieran cursos enfocados a la certificación, se atraería a nuevos estudiantes. Solo $20 \%$ dijo no tener planes de hacerlo.

Entre los comentarios generales resalta el de una estudiante quien asegura que disfrutó mucho su estancia y estaba impresionada con la variedad de clases que el departamento de español para extranjeros les ofrece. Si bien es imposible sacar conclusiones generales del testimonio de una persona, hay que decir que las diferentes opiniones de la comunidad apuntan a que la coordinación logra satisfacer las expectativas de los estudiantes.

\section{LOS ALUMNOS DE LA LEES}

Las respuestas de los 13 alumnos del programa de la LEES representa el $15.66 \%$ del total de la población. El $30.8 \%$ tiene una edad de 21 años, el otro $29.2 \%$ se sitúa entre los 22 y los 30 años. $76.9 \%$ de las respuestas fueron mujeres y $23.1 \%$ hombres.

Entre los aciertos que ubicamos se encuentra la difusión que se da tanto a la LEES como a los cursos para extranjeros. Docentes, alumnos y administrativos reconocieron que la difusión por medios electrónicos es amplia y detallada, como se muestra en la siguiente gráfica.
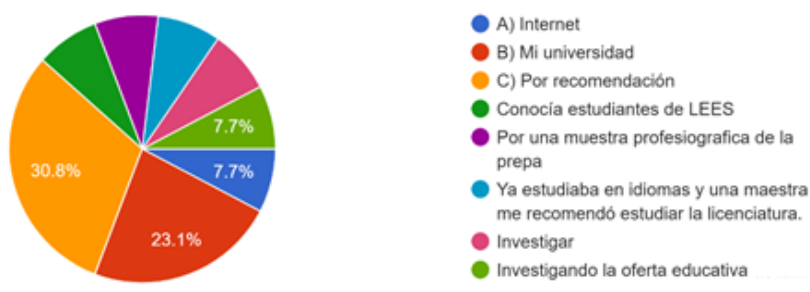

Gráfica 3. Difusión de la carrera

Entre las razones que tuvieron los estudiantes mexicanos para elegir estudiar la LEES encontramos que es la única universidad que oferta esta carrera dentro de sus posibilidades económicas y de movilidad para poder enseñar español fuera de México. Esto es debido a que se han dedicado al estudio de las lenguas y la licenciatura es una buena oportunidad, por seguridad en comparación con la ciudad de México y por la formación que el programa ofrece. 
6. ¿Consideras que el programa te está preparando para enfrentarte al campo laboral?

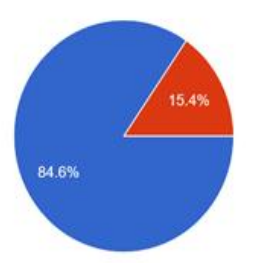

$$
\text { - Si }
$$

Gráfica 4. Preparación del egresado al campo laboral

Una fortaleza que se identificó es que los estudiantes de la LEES conocen al $100 \%$ su plan de estudios, la institución difunde la información de forma adecuada y los alumnos están informados sobre la trayectoria de sus estudios. Este aspecto es fundamental, pues al estar informados podrán sacar mejor provecho de las oportunidades que se les ofrecen durante la carrera. Los alumnos dijeron sentir empatía con sus profesores porque son amables y les enseñan cosas útiles. La mayoría se siente satisfecho en la relación alumno-docente, aunque como se muestra en la Gráfica 3 sólo el $84.6 \%$ siente que está preparado para enfrentarse al campo laboral. Este porcentaje podría cambiar, pues el programa de la LEES pasó recientemente por una reforma curricular, de tal forma que los alumnos que ingresaron en 2018 ya cursan este nuevo plan que contempla, entre otras cosas, mayor práctica docente previa al egreso, algo que se espera los prepare más al ámbito laboral.

Entre las sugerencias compartidas por los estudiantes de la LEES encontramos fortalecer el trabajo autónomo y reflexivo en las clases, incluir otras variantes del español como parte de los estudios y no dar prioridad a la variante española, más peso al estudio del sistema lingüístico del español y herramientas tecnológicas de vanguardia, así como incluir temas de cultura: literatura, historia de México, estudios de género, cine entre otros.

\section{DOCENTES Y ADMINISTRATIVOS}

En tanto que para los guiones se entrevistaron a cinco docentes por horas y de tiempo completo, que tienen desde un año hasta más de 30 años de experiencia docente, a las dos coordinadoras de la licenciatura y de los cursos de español que fungen como enlace entre las actividades académicas, así como administrativas y a dos directivos que se vinculan con los programas. Los perfiles de los docentes y los coordinadores son:

- Licenciada en filosofía, maestría en ELE.

- Cuatro Licenciados egresados de la LEES.

- Pasante de la LEES en proceso de titulación.

- Licenciada en psicología, maestría en el área y en educación, doctorado en inglés, docente de inglés y de español.
- Licenciada en diseño de interiores, maestría en tecnología educativa para la enseñanza del inglés.

Los docentes de la LEES señalaron que mantienen una relación positiva con los estudiantes y tratan de innovar en su enseñanza. La mayor dificultad que enfrentan es que todos los que entrevistamos eran docentes de tiempo completo o parcial, pero con cargo administrativo, lo que los lleva a tener una sobrecarga de actividades (situación muy típica actualmente en la mayoría de las universidades mexicanas), lo que no les permitía ofrecer la calidad que ellos deseaban en sus actividades docentes. Una forma en la que la administración trata de ayudar a los docentes es permitiéndoles que incluyan su carga administrativa como parte de sus horas laborales. En otros casos, el trabajo administrativo es remunerado de manera extra para el docente.

Por otro lado, los docentes entrevistados que dan clase a los estudiantes extranjeros carecen de experiencia, aunque cuentan con mucho entusiasmo para enseñar la lengua, lo que los lleva a innovar en las clases y a afrontar de mejor forma los obstáculos que se les presenten en beneficio de sus alumnos extranjeros. Uno de los problemas que ellos identificaron fue la falta de estructura organizacional que les impedía solicitar recursos tecnológicos de manera óptima, aunque expresaron sentirse apoyados por la coordinación.

Otro problema es la falta de libros institucionales, lo que los obliga a desarrollar sus propios materiales para cada clase. A decir de los entrevistados, existe un conjunto de materiales, elaborados por egresados y docentes, que bien podrían facilitar el trabajo académico en el aula, pero hasta la fecha nadie se ha dado a la tarea de recopilarlos y producirlos a nombre de la universidad. Habría que evaluar si vale la pena darse a la tarea de compilarlos mejores materiales para iniciar con la elaboración de materiales de la institución, sobre todo considerando que los materiales con que se cuenta en México son escasos. Otra posibilidad sería el diseño de materiales electrónicos, lo que brindaría mayor visibilidad a la institución no solo en México sino en el mundo.

También, se identificó una falta de estabilidad laboral, que no permite que los docentes, sobre todo los de hora clase, se sientan involucrados con la universidad, ni vean un compromiso a largo plazo con la institución; más bien, perciben el trabajo como algo transicional, lo que hace que no se sientan parte de la comunidad docente en la toma de decisiones ni de la planeación institucional. Este último punto se pudo corroborar porque algunos de ellos no eran conscientes de los derechos que tienen como docentes de la institución.

Sobre este aspecto, sería pertinente involucrar a los docentes por hora en las actividades académicas, así como darles una capacitación sobre los derechos laborales que tienen cuando ingresan a la institución y las oportunidades de crecimiento si 
deciden permanecer. Estas dos estrategias podrían ayudar a que los docentes más jóvenes quieran mantenerse en la institución, pues mantendrían un vínculo más fuerte y prevendría la fuga de capital humano ya formado. El programa carece de docentes preparados y no se han encontrado las estrategias adecuadas para fortalecer este aspecto. La política institucional pide que los docentes concursen de manera abierta por una plaza y algunos de los docentes que han logrado estas plazas por concurso han dimitido al poco tiempo, en detrimento del programa. Podría pensarse en un esquema más local, en el que se promueva la formación de los docentes novatos egresados del programa, para que al término de sus estudios se incorporen a la planta docente.

Finalmente, todos los sectores entrevistados identificaron como problema el aspecto de la inseguridad en México. Como se señaló, este asunto está fuera del alcance de la universidad, pues afecta no solo a esta institución sino a muchas otras en el país, lo que impide la llegada de más extranjeros al país. No obstante, la tecnología nos abre una opción de capacitación a distancia que podría ayudar a que la universidad capte extranjeros desde sus países de origen. Para lograr esto, se podría implementar un programa de educación a distancia para extranjeros.

\section{CONCLUSIONES}

La expansión acelerada por la que han pasado las universidades mexicanas en los últimos años hace imperante la necesidad de sondear áreas de oportunidad para la mejora del quehacer docente en términos de la enseñanza de español para extranjeros. Este proyecto intenta coadyuvar en la mejora de este proceso, al ver tanto dentro como fuera del aula, aquellas necesidades que requieren atenderse.

Para poder modernizar y mejorar la enseñanza, es importante conocer cuál es la percepción general de la población sobre el programa de español de la institución. La universidad no contaba con información sistematizada sobre los esfuerzos que los distintos actores implementan para tratar de mejorar la situación de la enseñanza del ELE y los problemas a los que se enfrentan. Este tipo de investigación es importante toda vez que permite ver los aciertos en cuestiones de enseñanza y aprendizaje de lenguas y las oportunidades que existen. Es así como este proyecto pretende contribuir al trabajo de investigación y trabajo docente que en la Universidad de Guanajuato se hace para hacer una evaluación informada y de esta manera llegar a la toma de decisiones pertinentes que forjen el camino a tomar en el futuro.

\section{REFERENCIAS}

[1] Centro Virtual Cervantes (2018) "Situación del español en el mundo". Recuperado de: http://www.cervantes.es/bibliotecas_documentacion_espanol/situacion _espanol_mundo/presentacion.htm.

[2] --------- (2020) "El español: una lengua viva. Informe 2020". Recuperado de: https://cvc.cervantes.es/lengua/espanol_lengua_viva/pdf/espanol_leng ua_viva_2020.pdf.

[3] Creswell, J. W. (2014) Research Design: Qualitative, Quantitative, and Mixed Methods. Sage.

[4] Dörnyei, Z. (2007). Research methods in applied linguistics. Oxford University Press.

[5] Edinumen (2021). https://edinumen.es/

[6] INEGI (2020) Migración. Recuperado de: https://www.inegi.org.mx/temas/migracion/.

[7] -------- (2007) Los extranjeros en México. México. Recuperado de: https://imumi.org/images/articulos/AgregadosDic/Proyectos/extranjero s_en_mexico_inegi_2001.pdf.

[8] Instituto Cervantes (2021) https://www.cervantes.es/default.htm

[9] Kaplan, R. B., \& Baldauf, R. B. (1997). Language Planning from Practice to Theory. Clevedon: Multilingual Matters.

[10] Pérez Velasco, M., \& González Freire, J. M. (2008). ¿Por qué estudiar español como lengua extranjera y no otra lengua? En XXI Foro de Especialistas Universitarios en Lenguas Extranjeras: Aprendizaje de Lenguas Extranjeras en Contextos Multiculturales e Indígenas. Tuxtla Gutiérrez: Universidad de Ciencias y Artes de Chiapas. Pp. 37-44. Recuperado

https://viancep2012.files.wordpress.com/2012/11/memoria-feule2008.pdf.

[11] Ruiz Zambrana, J. (2009). La situación actual de la lengua española en el mundo. Contribuciones a las Ciencias Sociales. Recuperado de: http://www.eumed.net/rev/cccss/05/jrz.htm.

[12] Suárez García, J. (2021) todoELE. http://todoele.es/home

[13] Tollefson, J. (1994) Planning Language, Planning Inequalities. London: Longman.

[14] Universidad Autónoma de Querétaro (2020). "Cursos de Español para Extranjeros (Spanish Course)". Recuperado de: https://fll.uaq.mx/index.php/cursos-extra.

[15] Universidad de Guanajuato (2019). Plan de Desarrollo Institucional 2010-2020. Guanajuato. Universidad de Guanajuato. Recuperado de: https://www.superprof.mx/blog/estudia-espanol-mexico/

[16] Universidad de Guadalajara (2020) "Programa de Español para Extranjeros (PEPE)". Recuperado de: http://www.cgci.udg.mx/pepe/

[17] Universidad Nacional Autónoma de México (2020). "Centro de Español para Extranjeros (CEPE)". Recuperado de: https://www.cepe.unam.mx/

[18] Universidad Veracruzana (2020) "Escuela para Estudiantes Extranjeros (EEE). Recuperado de: https://www.uv.mx/eee/ 